\title{
Anesthetic Challenges of a Child with Sturge-Weber Syndrome for Epilepsy Surgery: A Case Report
}

\author{
Roshan Kurian $^{1} \quad$ Karen R. Lionel ${ }^{1} \quad$ Ramamani Mariappan ${ }^{1}$
}

Address for correspondence Karen R. Lionel, MD, DM (Neuroanaesthesia), Department of Anaesthesia, Christian Medical College, Vellore, Tamil Nadu 632002, India (e-mail: karen@cmcvellore.ac.in).
Abstract
Keywords
- Sturge-Weber syndrome
- epilepsy surgery
- anesthetic implications

Sturge-Weber syndrome is a rare congenital disease, also called encephalotrigeminal angiomatosis, caused by persistence of transitory primordial arteriovenous connections of the fetal intracranial vasculature. It is characterized by vascular malformations with capillary venous angiomas that involve the face, choroid of the eye, and leptomeninges. The main clinical features of this syndrome are port-wine stains, glaucoma, convulsions, and angiomas of the airway. Anesthesia management is directed toward anticipating a difficult airway, avoiding trauma to the hemangioma during airway manipulation, preventing the rise in the intracranial and intraocular pressures, anticipating and managing massive blood loss and the complications associated with massive blood transfusion, and avoiding factors that might trigger a seizure such as hypoxia, hypercarbia, hypotension, hypoglycemia, and hyperthermia.

\section{Introduction}

Sturge-Weber syndrome (SWS), also called encephalotrigeminal angiomatosis, is a rare congenital disorder that occurs sporadically. The pathognomonic manifestations of this neurocutaneous disorder are leptomeningeal angiomas extending into the cerebral cortex and intracranial calcification of this lesion, along with ipsilateral angiomatous lesions involving the facial skin, typically in the ophthalmic (V1) and maxillary (V2) distributions of the trigeminal nerve. ${ }^{1}$ It often presents with mental retardation, hemiparesis, or refractory epilepsy. The most recognizable clinical feature of SWS is the facial angioma or port wine stain, which has been reported to occur in up to $70 \%$ of patients. ${ }^{2}$ Vascular angiomas may involve the mouth and airway and can lead to difficult mask ventilation, laryngoscopy, and intubation. Cardiac anomalies, which may be associated with this syndrome include septal defects, valvular stenosis, great vessels transposition, and deep arteriovenous malformation (rare), which may lead to considerable shunt and cardiac hypertrophy and failure. In this case report, we would like to highlight the anesthetic challenges of a child with SWS coming for epilepsy surgery.

\section{Case Report}

We report a case of a 2.3-month-old, 9-kg, child with SWS who presented with developmental delay and left-sided refractory focal motor seizures. On examination, she had the typical cutaneous manifestations of SWS characterized by a large capillary angiomatous lesion involving the entire right scalp, forehead, face, neck, and upper chest till the nipple region with an abrupt cutoff at the midline. A magnetic resonance imaging (MRI) of the brain showed diffuse thinning and atrophy of the right cerebral hemisphere with marked leptomeningeal enhancements. There was compensatory enlargement of the left hemisphere ( $\boldsymbol{- \text { Fig. }}$ 1). Though a complete airway examination was not possible preoperatively to know the extent of the lesion, we had anticipated the airway involvement because of extensive cutaneous involvement. Airway involvement was also confirmed by her mother's history where she revealed that the angiomatous lesions involved the posterior pharyngeal wall, which was visible when the child cried. But, there was no history suggestive of airway obstruction. Routine blood investigations,

\section{received}

July 11,2018

accepted after revision

August 22, 2018

published online

January 25, 2019
DOI https://doi.org/

10.1055/s-0038-1675892

ISSN 2348-0548.
Copyright $\odot 2019$ Indian Society of Neuroanaesthesiology and Critical Care
License terms

(ㅇ) (1) $\Theta \circledast$ 


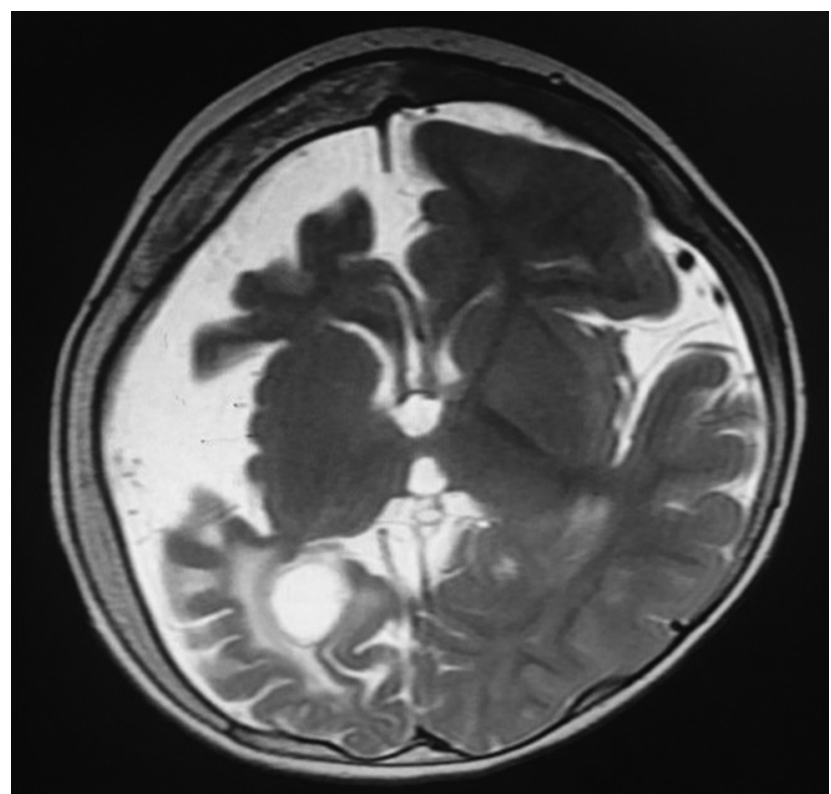

Fig. 1 MRI brain (T2W image, axial cut) showing diffuse thinning and atrophy of the right cerebral hemisphere with marked leptomeningeal enhancements (leptomeningeal angiomatosis) and compensatory enlargement of the left hemisphere. MRI, magnetic resonance imaging; T2W, T2-weighted.

electrocardiogram (ECG), and chest X-ray were within normal limits. She was scheduled for anterior temporal lobectomy (ATL), amygdalohippocampectomy (AH), and hemispherotomy. The child was pre-medicated with Syrup trichlorofos at $75 \mathrm{mg} / \mathrm{kg} 1$ hour prior to induction.

The child was asleep while wheeling into the theater, thus a steal induction was successfully performed with incremental rising concentrations of sevoflurane. Apart from the routine monitors at induction, which were pulse oximetry, ECG, non-invasive blood pressure, and an end-tidal carbon dioxide $\left(\mathrm{ETCO}_{2}\right)$, other monitoring such as an invasive arterial line in the right radial artery, minimum alveolar concentration of the inhalational agent, neuromuscular monitor with a nerve stimulator, bispectral (BIS) monitor, temperature, and urine output all were monitored during surgery. As soon as the IV access was established, sevoflurane was switched off, and anesthesia was maintained with total intravenous anesthesia. Since there was a significant lesion on the airway mucosa, and to avoid trauma associated with a blind routine laryngoscopy and study the extent of the lesion, we opted for a videolaryngoscopy- (C-MAC, Video Laryngoscope, KARL STORZ, Germany) guided intubation, which revealed extensive airway involvement extending up to the vocal cords ( - Fig. 2). The airway was secured with a 5-size uncuffed endotracheal tube (ETT) fixed at $12 \mathrm{~cm}$. Anesthesia was maintained with propofol infusion at 150 to $200 \mu \mathrm{g} / \mathrm{kg} / \mathrm{min}$, which was titrated to keep the BIS of 40 to 50 , injection of fentanyl infusion at 1 to $2 \mu \mathrm{g} / \mathrm{kg} / \mathrm{h}$, and an injection of atracurium infusion of 0.3 to $0.5 \mathrm{mg} / \mathrm{kg} / \mathrm{h}$ titrated to achieve two twitches on the train of four. Since there was capillary hemangiomatous lesion involving the right side of

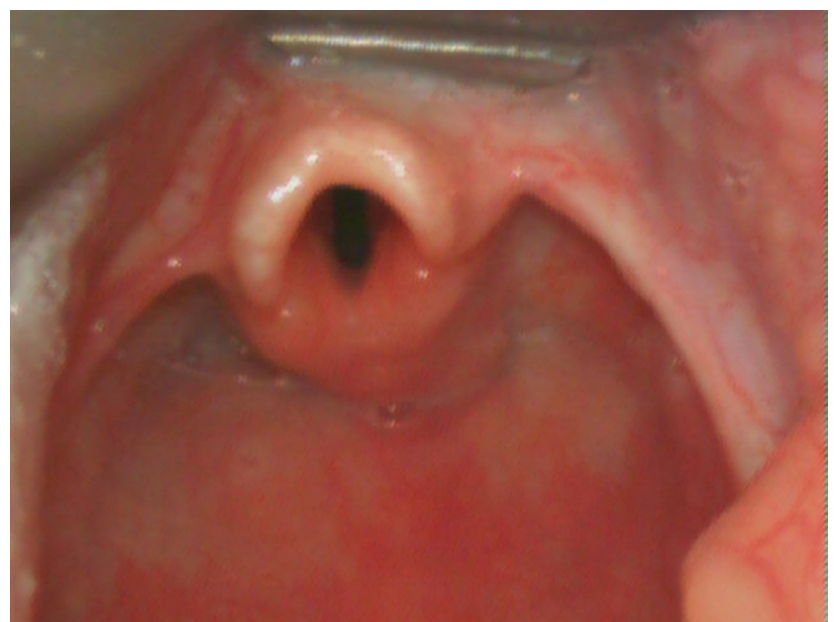

Fig. 2 Videolaryngoscopic image showing extensive hemangiomatous lesions involving the oropharynx and larynx.

neck and chest, the left internal jugular vein was cannulated, and a 5.5-F triple lumen catheter was inserted.

Intraoperatively from the commencement of skin incision to the exposure of dura, there was a continuous, persistent blood loss due to extensive skin and bone involvement. Anticipating the risk of hemorrhage, we gave a loading dose of tranexamic acid at $20 \mathrm{mg} / \mathrm{kg}$ before the skin incision, followed by an infusion at $1 \mathrm{mg} / \mathrm{kg} / \mathrm{h}$ till skin closure. Even before hemispherotomy was initiated, the hemoglobin had dropped from 11.4 to $8.5 \mathrm{~g} \%$, which was replaced with crystalloids and packed red cells. The dural, as well as the intracranial anomalous brain tissue, was strewn with thin abnormal capillary malformations, which resulted in a total loss of $650 \mathrm{~mL}$, which was almost his total blood volume despite immaculate dissection and resection by the senior surgeon. This blood loss was replaced with $60 \mathrm{~mL}$ of cryoprecipitate, $150 \mathrm{~mL}$ of fresh frozen plasma, and $300 \mathrm{~mL}$ of packed red cells. The child remained warm and hemodynamically stable throughout the surgery, and the arterial blood gas that was done at skin closure was within normal limits. Since it was a long duration surgery (6 hours) associated with massive transfusion and the airway involvement by the lesion, we had decided to electively ventilate the child overnight. The child was sedated and ventilated with midazolam and fentanyl infusion overnight and got extubated the next day. She was discharged seizure free on the 10th postoperative day.

\section{Discussion}

The Sturge-Weber syndrome, also known as encephalotrigeminal angiomatosis, is characterized by congenital angiomas and involves the skin over the trigeminal distribution. Sometimes, these vascular changes can also be found in the dura, leptomeninges, brain tissue, and pituitary gland. No pattern of inheritance is generally noted. Angiomas are frequently unilateral with faciotrigeminal distribution; sometimes these can be bilateral. In some cases, angiomas are found over the truncal and extremities 
areas and in deeper structures such as the thymus, lung, spleen, and lymph nodes., ${ }^{3,4}$ SWS may involve the mucosa of the nose, palate, gingiva, tongue, larynx, and trachea posing a challenge to laryngoscopy and intubation. ${ }^{5}$ This involvement can be diagnosed in the preoperative MRI, if slices involved the airway as well. Anesthesia-induced vasodilation increases the size of the lesion can lead to worsening of airway obstruction, and even a mild injury to these lesions during airway manipulation may result in uncontrolled hemorrhage. Therefore, intubation should be done using a soft, well-lubricated, non-stylet cuffed ETT, and careful oropharyngeal and tracheobronchial suction are crucial in avoiding trauma to these lesions. ${ }^{6,7}$

In our case, one of the major anesthetic concern was an anticipated difficult airway because of lesions involving the oral cavity and pharynx. We performed a gentle laryngoscopy using a C-MAC video laryngoscope, which enabled us to gauge the extent of airway involvement and ensured that the intubation was atraumatic. Furthermore, raised intracranial pressures due to the cerebral edema associated with the refractory seizures and raised intraocular pressure are commonly seen in patients with SWS attributed to pre-existing glaucoma to require a smooth induction and intubation to limit the rise in both, which was achieved with video laryngoscope intubation. A fiberoptic bronchoscopic intubation could have also been an acceptable option to secure the airway with the advantage of being able to quantify the airway involvement below the level of the vocal cords.

The second major concern was massive blood loss and its associated complication. Since we had anticipated massive blood loss and adequate blood was arranged pre-operatively, blood and blood products were transfused appropriately at the correct time without making the child going into the phase of dilutional coagulopathy as well as fluid overload.

Since the child was having refractory epilepsy, factors that can precipitate perioperative seizures, such as hypoglycemia, hypotension, hypoxemia, and hyperthermia, were monitored for and avoided. Additionally, since the child had been on four antiepileptic agents, the enzyme induction caused by the anticonvulsants would definitely influence the metabolism of the muscle relaxants and the anesthetic requirements as well. To prevent an overdosage and to prevent awareness, we used a BIS monitor to titrate the anesthetic and a neuromuscular monitor to titrate the relaxant infusion.

\section{Conclusion}

We conclude that patients with SWS should be carefully evaluated for associated anomalies involving the airway, cardiovascular system, and ophthalmic involvement as well. The perioperative anesthetic management should be planned in a way to avoid trauma to the hemangiomatous lesions involving the airway, preventing any rise in intraocular and intracranial pressure, prevention and management of massive blood loss, as well avoidance of factors that may trigger a seizure. A careful plan must be made regarding a smooth induction and intubation and a diligent extubation.

\section{Funding}

None.

\section{Conflict of Interest}

None declared.

\section{References}

1 Roach ES. Neurocutaneous syndromes. Pediatr Clin North Am 1992;39(4):591-620

2 Thomas-Sohl KA, Vaslow DF, Maria BL. Sturge-Weber syndrome: a review. Pediatr Neurol 2004;30(5):303-310

3 Schmitt J. Visceral aspects of the phakomatosis. In: Vinken PJ, Bruyn GW, eds. Handbook of Clinical Neurology, volume 14. Amsterda North Holland Publishing Co.; 1972: 668-730

4 Alexander GL. Sturge-Weber syndrome. In: Vinken PJ, Bruyn GW, eds. Handbook of Clinical Neurology, volume 14. Amsterda North Holland Publ. Co.; 1972:223-240

5 Wong HS, Abdul Rahman R, Choo SY, Yahya N. Sturge-Weber-Syndrome with extreme ocular manifestation and rare association of upper airway angioma with anticipated difficult airway. Med J Malaysia 2012;67(4):435-437

6 Butler MG, Hayes BG, Hathaway MM, Begleiter ML. Specific genetic diseases at risk for sedation/anesthesia complications. Anesth Analg 2000;91(4):837-855

7 Batra RK, Gulaya V, Madan R, Trikha A. Anaesthesia and the Sturge-Weber syndrome. Can J Anaesth 1994;41(2):133-136 